\title{
Development of Circadian Sleep-Wake Rhythms in Preterm and Full-Term Infants
}

\author{
I. CAROLINE MCMILLEN, JOYCE S. M. KOK, T. MICHAEL ADAMSON, JAN M. DEAYTON, \\ AND RACHEL NOWAK
}

Department of Physiology, Monash University [I.C.M., J.S.M.K., J.M.D., R.N.] and Department of Paediatrics, Monash Medical Centre [T.M.A.], Clayton, Victoria, 3168, Australia

\begin{abstract}
We have compared the roles of neurologic maturity and environmental time cues in the development of the entrained circadian sleep-wake rhythm in the preterm and term human infant. The preterm infants $(n=19)$ spent some time after birth in a hospital nursery with no environmental time cues, whereas the term infants $(n=$ 22) were exposed from birth to a cyclical light and dark environment with one major caregiver. The circadian sleepwake rhythm in the preterm infants entrained after a similar time of exposure to an environment with daily time cues but at an earlier postconceptional age when compared with the term group. We conclude, therefore, that it is the length of exposure to environmental time cues, rather than neurologic maturity, that determines the entrainment of the circadian rhythm of sleep and wakefulness in the human infant. (Pediatr Res 29: 381-384, 1991)
\end{abstract}

The daily sleep-wake cycle in the adult is a circadian rhythm, i.e. it is a behavioral rhythm that is generated by an endogenous biologic clock and in the absence of external time cues it "freeruns" with a periodicity of approximately $25 \mathrm{~h}$ (1). Normally, however, the circadian sleep-wake pattern is entrained or synchronized to an exact 24 -h period by external time cues that are known as zeitgebers. It has been proposed that the light-dark cycle and also social cues act as zeitgebers on the endogenous circadian pacemaker to synchronize the human sleep-wake cycle to a 24 -h period (1).

Although there is extensive literature that documents that sleep is consolidated toward the night by the 6th to 14th wk of life (26), there have been few attempts to define the factors that determine the timing of entrainment of the infant's circadian sleep-wake rhythm to the day-night cycle. It has been widely accepted that the emergence of an entrained circadian sleepwake pattern in the human infant is predominantly the result of maturation of the CNS (2-6). This is supported by the demonstration using spectral analysis that sleep-wake activity has a more prominent $24-\mathrm{h}$ component in term infants when compared with preterm infants at the same postnatal age $(7-13 \mathrm{wk})$ (7).

It may be misleading, however, to compare the development of circadian sleep-wake rhythms in pre- and full-term infants without taking into account the differences in the range of zeitgebers that these infants are exposed to after birth. A fullterm infant born at home or in hospital, where "rooming-in" with the mother is the normal practice, is exposed to a cyclical

Received May 28, 1990; accepted November 12, 1990

Correspondence and reprint requests: Dr. I. C. McMillen, Department of Physiology, Monash University, Clayton, Victoria 3168, Australia.

Supported by the Sudden Infant Death Research Foundation (Victoria, Australia), the Clive and Vera Ramaciotti Foundation, and the Victorian Health Promotion Foundation. light-dark environment and the circadian behavioral and social patterns of a single caregiver. In contrast, the preterm infant will spend some time after birth in a hospital neonatal nursery where there is constant illumination and where there are several different caregivers in any $24-h$ period (8). Any difference between term and preterm infants in the timing of the development of an entrained circadian sleep-wake pattern may therefore not only be due to differences in maturity but also to exposure to a different range of zeitgebers in the immediate postnatal period. We have argued that if neurologic maturity were critical for the emergence of an entrained circadian sleep-wake rhythm, then this would be reflected in the emergence of an entrained rhythm at a similar postconceptional age in preterm and term infants. If, however, exposure to zeitgebers such as the daily light-dark cycle were important, then entrainment of the sleep-wake rhythm would occur in preterm and term infants after a similar time of exposure in the home environment.

We have therefore determined the timing of the emergence of an entrained sleep-wake rhythm in preterm and term infants and investigated the relationship between time to entrainment and postconceptional age and the length of exposure to a home environment. We have defined the home environment as a cyclical light-dark environment with a single caregiver, and this includes the rooming-in of the term infant with the mother in the hospital.

\section{SUBJECTS AND METHODS}

Infants. Ethical approval for this study was granted by the Monash University Standing Committee on Ethics and Human Experimentation. Twenty-two term (gestational age 38-42 wk; 13 male, nine female) and 19 preterm (gestational age 27-35 wk; nine male, 10 female) infants were recruited.

In the term group, 18 infants were delivered spontaneously, three after induced labor and one by cesarean section. The range of birth weights was 2.8 to $4.6 \mathrm{~kg}$ and the range of Apgar scores at 1 and $5 \mathrm{~min}$ was 8 to 10 . All term infants were breast-fed on demand for 4 to 6 mo after birth. The postconceptional age of the term infants was calculated from the first day of the last menstrual period and on the premise that the expected date of delivery was $40 \mathrm{wk}$ after this date.

All preterm infants were recruited in the Kate Campbell Neonatal Nursery at the Monash Medical Centre (Clayton, Victoria, Australia). In this preterm group, seven infants were delivered spontaneously, five after induced labor and seven by cesarean section. The range of birth weights of the preterm infants was 1.0 to $2.4 \mathrm{~kg}$ (median $=1.84 \mathrm{~kg}$ ) and the range of Apgar scores at 1 min was 0 to 10 (median $=8$ ) and at $5 \mathrm{~min}, 3$ to 10 (median $=9$ ). Infants in the preterm group had a history of respiratory distress $(n=17)$, neonatal jaundice $(n=15)$, patent ductus arteriosus $(n=3)$, and hypotension $(n=4)$. At the time of recruitment (between 33 and $39 \mathrm{wk}$ postconceptional age), no infant had respiratory or neurologic problems. Two of the pre- 
term infants were primarily breast-fed, 12 were primarily formula-fed, and five were both breast- and formula-fed during the 6 mo of the study. The postconceptional age of the preterm infants was calculated by the addition of the infant's gestational age (derived from the date of the mother's last menstrual period and confirmed by ultrasound in the 1st trimester) and postnatal age.

Sleep-wake recordings. A 24-h sleep-wake activity profile of each infant was recorded at 1 to $2 \mathrm{wk}$ intervals from the time of recruitment until the infants reached a postconceptional age of 60 wk.

In the neonatal nursery, the 24-h sleep-wake activity profile of each preterm infant was recorded at 1 to 2 wk intervals until the infant was discharged home. At home, the 24-h sleep-wake activity profiles of the preterm and term infants were recorded every $2 \mathrm{wk}$ by the major caregiver (usually the mother) on diary charts provided. The 24 -h sleep-wake record was made between $1200 \mathrm{~h}$ and $1200 \mathrm{~h}$ of the following day, the times at which the infant awoke or went to sleep were recorded on the chart, and the period of time spent asleep by the infant was indicated by filling in the corresponding period on the time axis (Fig. 1). To ensure consistency between recordings made by different mothers, infant waking was defined as eyes open with accompanying noise and movements.

Light intensity. Light intensity was measured during the day and night in the neonatal nursery and in the home of all preterm infants using a light meter (Lunnasix 3, Gossen Company, Germany) with a scale range of $1-22$. The reading was converted to lux (lumen $/ \mathrm{m}^{2}$ ) units and logarithmically transformed because the human eye responds to changes in light intensity in a logarithmic fashion (9). Although the light intensity was not measured in the homes of term infants, the light intensity during nighttime feedings was recorded by the mothers as either dark, dim, or full lights throughout the course of the study.

Analysis of records. For each infant, the 24-h sleep-wake pattern in any recording period was mapped twice side by side to determine the emergence of an entrained circadian pattern of sleep-wake activity (see Fig. 1). This method of "double-plotting" sleep-wake activity is commonly used because it aids in the identification of free-running and entrained patterns of circadian behavior (2). The time of entrainment was defined as when the majority of the sleep episodes (including the longest sleep bout) were consolidated toward the dark phase of the day-night cycle and the time of onset and offset of sleep were consistent (within $1 \mathrm{~h}$ ) between preceding and subsequent recording periods. Two

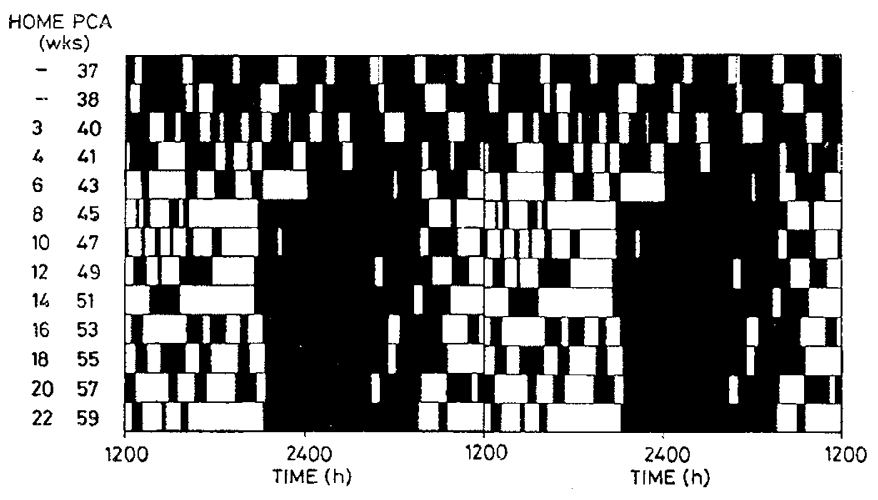

Fig. 1. Graph representation of the 24-h sleep-wake activity of infant A during the first 4 to 6 mo after birth. The black bars represent the period of time when the infant was asleep, and the 24-h pattern in any recording period is mapped twice, side by side, to generate a double-plot (8). The postconceptional age (PCA) of the infant and the length of exposure of the infant to the home environment (HOME) at any recording time is given on the left of the circadian double-plot. Infant A was a female, born at $34 \mathrm{wk}$ gestation and discharged home at a postconceptional age of $38 \mathrm{wk}$. The circadian sleep-wake activity rhythm in this infant entrained after $8 \mathrm{wk}$ in the home environment. observers assessed all circadian double-plots independently and each made an estimate of the recording period in which circadian entrainment was defined. The most conservative estimate of time of entrainment, i.e. the date of the latest recording period, was then used. Using the criteria defined above, we find that independent observers agree on the time of entrainment $( \pm 1$ recording period) in $75 \%$ of cases. The time of entrainment of sleep-wake activity was expressed in relation to the infant's postconceptional age and postnatal age (weeks after birth) and also in relation to the length of time that the infant had spent in the home (which included rooming-in) environment.

Statistical analysis. All results are expressed as mean \pm SD. A two-way analysis of variance with replication and with place (i.e. hospital or home) and time (i.e. day or night) as the specified variables was used to analyze the light intensity data (after logarithmic transformation). Regression analysis was used to correlate the gestational ages of the infants at birth with the time taken for an entrained sleep-wake pattern to emerge, defined in terms of either postconceptional age, postnatal age, or time at home. The mean time to entrainment in the preterm and term groups was compared using the unpaired $t$ test. The cumulative frequency distributions of the time to entrainment in the preterm and term groups of infants were compared using the Kolmogorov-Smirnov two-tailed test.

\section{RESULTS}

Light intensity. In the neonatal nursery, the mean intensity of light during the day (6210 lux) or night (1566 lux) was significantly higher $(p<0.001)$ than the mean intensity of light during the day (616 lux) or night ( 9 lux) in the homes of the preterm infants. In the hospital, the change in the mean intensity of light from day to night corresponded to a change from bright to normal light, whereas at home the difference in mean light intensity from day to night corresponded to a change from normal light to dark (9).

Entrainment of sleep-wake activity. In the group of preterm infants, there was a significant inverse correlation $(r=0.56, p<$ 0.05 ) between gestational age and the postnatal age at which entrainment of the circadian sleep-wake pattern occurred and also between gestational age and the length of time spent in the hospital neonatal nursery $(r=0.92, p<0.05)$ (Fig. 2). The postnatal delay in entrainment in preterm infants born at a younger gestational age may therefore have been a consequence of the longer time spent in the nursery and a delay in exposure to the zeitgebers in the home environment (Fig. 2).

The suggestion that exposure to such zeitgebers may play a major role in entrainment is supported by the observation that the circadian sleep-wake rhythm entrained after a similar mean time of exposure to the home environment in the preterm $(9.8 \pm 2.2 \mathrm{wk})$ and term $(8.7 \pm 2.7 \mathrm{wk})$ groups. Further, when the data from the preterm and term infants were combined, there was no inverse relationship between the postconceptional age on exposure to the home environment and the time required for an entrained circadian sleep-wake pattern to emerge (Fig. 3). Therefore, although the preterm infants were exposed to the home environment at an earlier age than the term group, the time taken for entrainment to occur was similar in both groups. Consequently, we found that the circadian sleep-wake pattern entrained at a significantly earlier mean postconceptional age in the preterm infants $(47.0 \pm 2.2 \mathrm{wk})$ when compared with the term group $(48.9 \pm 2.7 \mathrm{wk}, p<0.05)$.

The cumulative frequency distribution of the entrainment patterns in the two groups of infants were also significantly different $(p<0.01)$ when the time of entrainment was expressed in terms of postconceptional age (Fig. 4). Although an entrained sleep-wake pattern had emerged in $50 \%$ of the preterm infants by a postconceptional age of $47 \mathrm{wk}$, only one of the term infants had entrained by this age (Fig. $4 A$ ). The cumulative frequency distributions of the entrainment patterns in the two groups of 


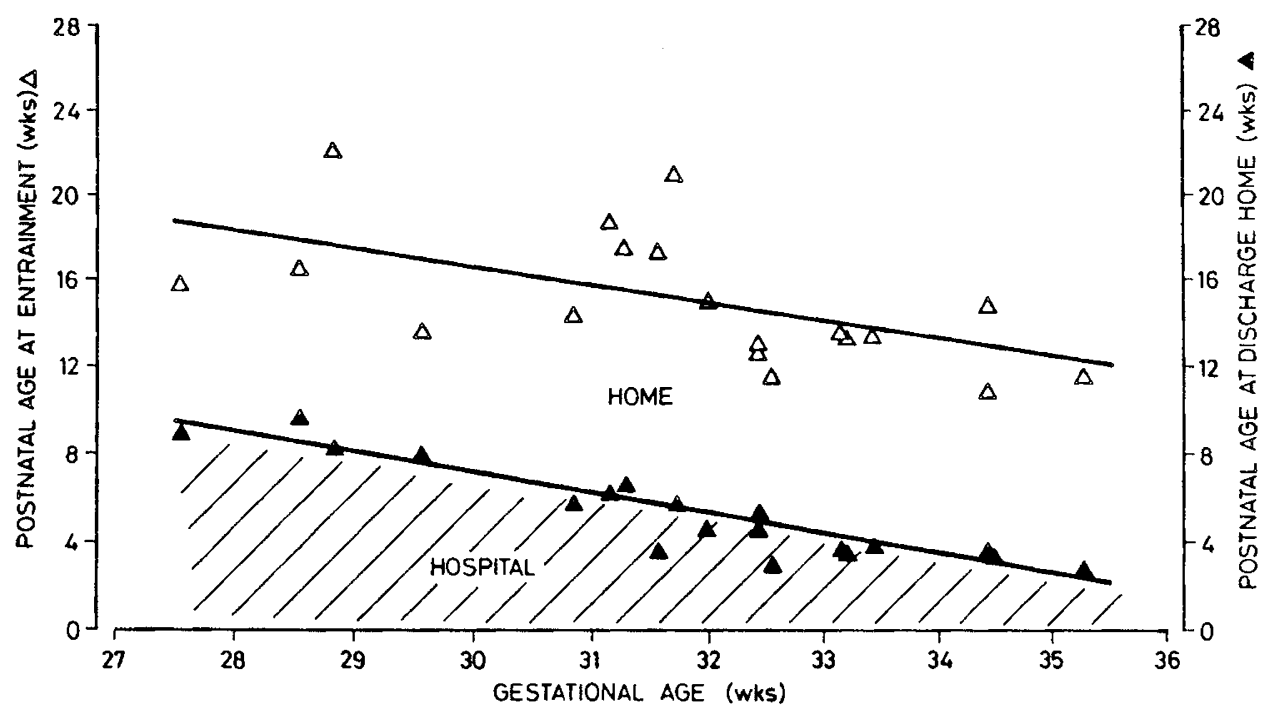

Fig. 2. The relationship between the gestational age of the preterm infants and the postnatal age at which the infants were discharged into the home environment $(\Delta)$ and also the postnatal age at which an entrained circadian rhythm of sleep and wakefulness emerged $(\Delta)$.

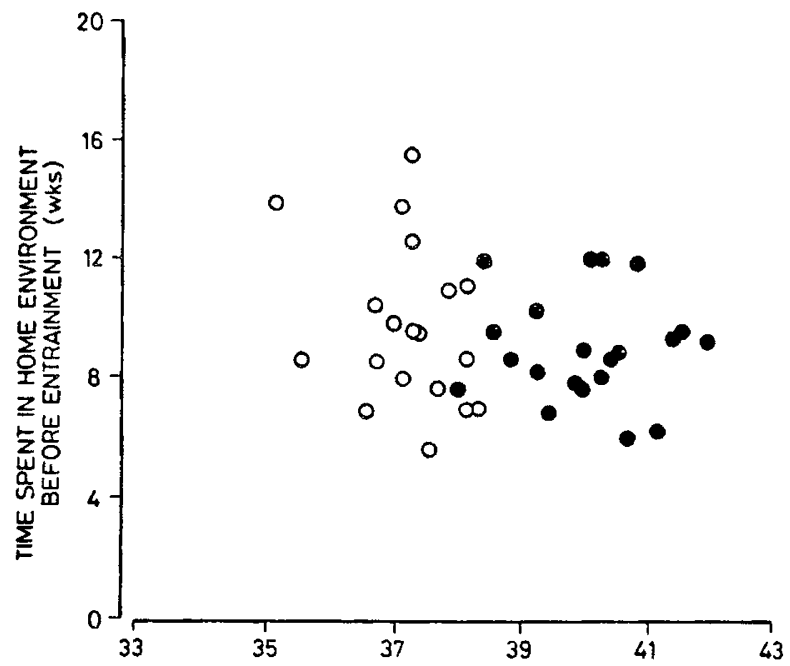

POSTCONCEPTIONAL AGE ON EXPOSURE TO HOME ENVIRONMENT (wks)

Fig. 3. The length of time spent in the home environment (which includes the rooming-in situation) before the emergence of an entrained circadian sleep-wake rhythm in each infant plotted against the postconceptional age of the preterm $(O)$ and the term $(\bullet)$ infants on first exposure to the home environment.

infants, when expressed in terms of the time spent in a home environment, were not significantly different (Fig. $4 B$ ).

\section{DISCUSSION}

It would appear from this study that the length of exposure to an environment with a single caregiver and light-dark cycle is the major determinant in the timing of the emergence of a zircadian sleep-wake pattern in the human infant. It would also appear that the postconceptional age of the infant is less critical $n$ the timing of entrainment of sleep-wake activity.

It is important to note, however, that all preterm infants in his study were discharged into the home environment at a ostconceptional age of at least $35 \mathrm{wk}$, and it may be that the ate of entrainment of the sleep-wake rhythm in infants exposed o a home environment before $35 \mathrm{wk}$ is not comparable with hose in the present study. Although postconceptional age did lot appear to be of major importance in entrainment, it was nteresting that in this study the infants who took longer to
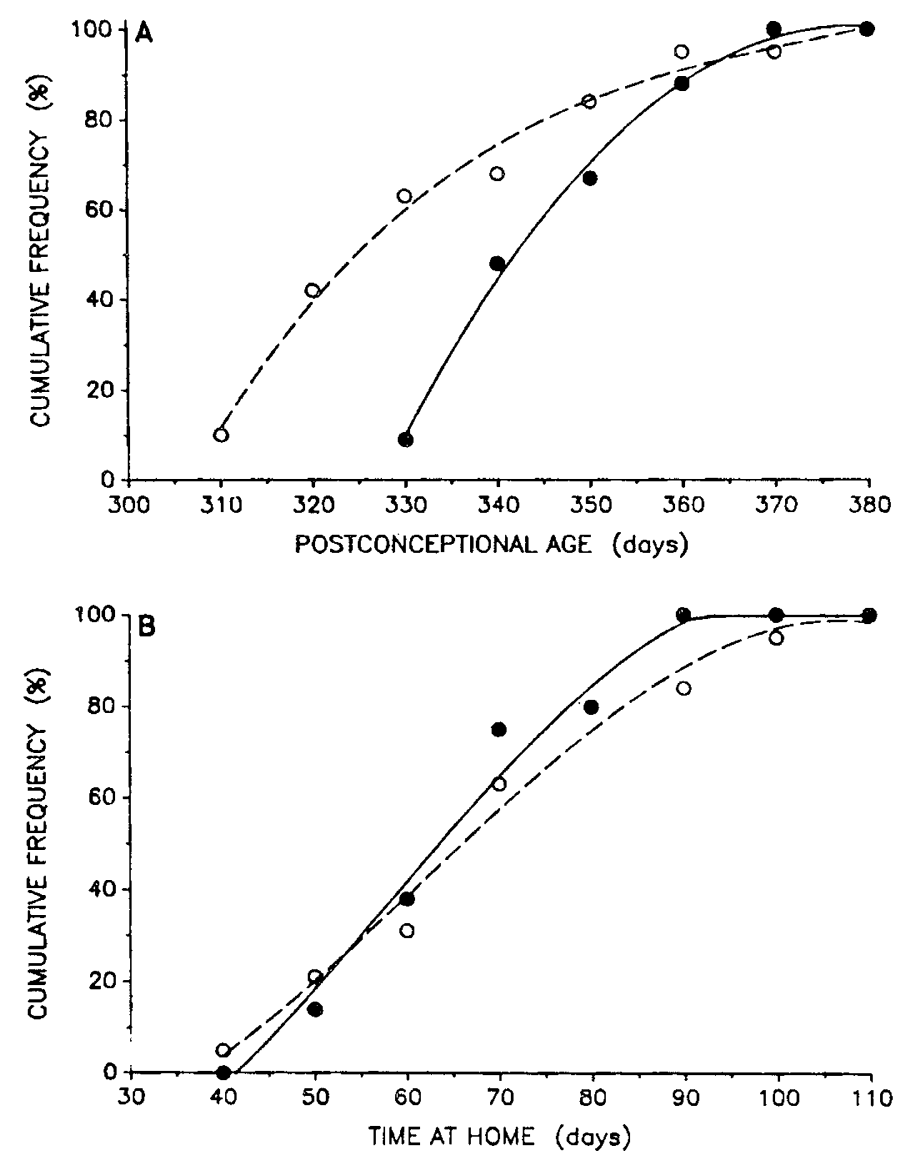

Fig. 4. The proportion of the total number of preterm $(O)$ and term $(\bullet)$ infants that had developed an entrained circadian sleep-wake rhythm by the postconceptional age indicated $(A)$ or after the designated time spent in the home environment $(B)$.

entrain (i.e. more than $13 \mathrm{wk}$ after discharge home) were all in the preterm group.

The emergence of an entrained circadian pattern of sleep-wake activity in the human infant requires that the afferent and efferent neural pathways to and from the hypothalamic circadian pacemaker are present and that the circadian clock is functional. Our findings would suggest that these components of the circadian 
rhythm generating system are in place as early as $35 \mathrm{wk}$ postconception and that the rate-limiting step in the emergence of an entrained behavioral rhythm after this age is the length of exposure to cyclical zeitgebers.

Our study has not identified the major zeitgeber present in the home environment. It may be relevant that we had to exclude one term infant from the statistical analysis because this infant never developed an entrained circadian sleep-wake pattern-this infant was also the only infant that was fed at night in full lights throughout the entire study period. This could indicate the importance of a normal light-dark cycle in the entrainment of the biologic clock.

Alternatively, circadian information could be conveyed by the single caregiver in the home environment through either her circadian patterns of behavior or through feeding. In the present study, all the term infants were breast-fed on demand for at least the first 4 mo of the study, whereas most of the preterm infants were fed either a cow's milk formula only or both cow's milk formula and breast milk. Inasmuch as entrainment of the sleepwake cycle occurred in the preterm and term infants after a similar time of exposure to the home environment despite the different feeding regimes, it would appear that breast feeding per se may not be the major zeitgeber in this environment.

In a previous study, Sander et al. (10) found that the ability of an infant to differentiate between day and night with regard to sleep and wakefulness was already evident by the 4 th $d$ of life in infants that received individual caretaking from birth. In contrast, the ability to recognize day and night was not evident in a group of infants reared in a hospital nursery by multiple caregivers until the 11 th postnatal day, when the infants were placed with a single caregiver. The relative roles of the cyclic light-dark environment and the single caregiver in the entrainment of circadian behavior in the infant remain to be determined.

The findings of our study may be important in the definition of an optimal environment for neonatal intensive care. Our results would suggest that exposure of preterm infants in a hospital nursery to a cyclical light-dark environment would result in earlier synchronization of the infants behavioral and hormonal rhythms with the external environment. In a recent study, preterm infants in hospital were placed in either a cyclical light-dark or a continuously lit environment for at least $10 \mathrm{~d}$ before dis- charge home (11). At 6 and $12 \mathrm{wk}$ after their expected date of delivery, infants who had stayed in the light-dark environment had gained more weight and slept more when compared with the control infants. Whether these changes were related to an earlier onset of an entrained circadian sleep-wake rhythm was not established.

In conclusion, our results suggest that external zeitgebers play a greater role in the emergence of the circadian sleep-wake rhythm in the newborn infant than has been previously considered. Such advances in our understanding of the physiologic control of sleep during the first 2 to 3 mo of life may lead to a better understanding of those pathologic events that may occur during sleep in infancy.

Acknowledgments. The authors thank the nursing and medical staff of the neonatal unit for their cooperation with this study and also the Nursing Mothers Association (Victoria) for their assistance. We thank David Caddy for his statistical advice.

\section{REFERENCES}

1. Wever RA 1986 Characteristics of circadian rhythms in human functions. Neural Transm [Suppl] 21:323-373

2. Kleitman N, Engelman TB 1953 Sleep characteristics of infants. J Appl Physiol 6:269-282

3. Parmalee Jr A, Wenner W, Schulz H 1964 Infant sleep patterns from birth to 16 weeks of age. J Pediatr 65:576-582

4. Coons S, Guilleminault C 1982 Development of sleep-wake pattern and nonrapid eye movement sleep stages during the first six months of life in normal infants. Pediatrics 69:390-396

5. Alley JM, Rogers CS 1986 Sleep patterns of breast-fed and non-breast fed infants. Pediatr Nurs $12: 349-351$

6. Navelet $Y$, Benoit $O$, Bouard G 1982 Nocturnal sleep organisation during the first months of life. Electroencephalogr Clin Neurophysiol 54:71-78

7. Hellbrugge T 1974 The development of circadian and ultradian rhythms of premature and full term infants. In: Schevring LS, Halberg F, Pauly JE (eds) Chronobiology. Igaku Shoin, Tokyo, pp 339-341

8. Blackburn S 1982 The neonatal ICU: a high-risk environment. Am J Nurs $82: 1708-1712$

9. Hopkinson RG 1969 Basic lighting principles and terminology. In: Lighting and Seeing. William Heinman Medical Books Ltd., London, p 54

10. Sander LW, Julie HL, Stechler G, Burns P 1972 Continuous 24-hour interactional monitoring in infants reared in two caretaking environments. Psychosom Med 34:270-282

11. Mann NP, Haddow R, Stokes L, Goodley S, Rutter N 1986 Effect of night and day on preterm infants in a newborn nursery: randomised trial. $\mathrm{Br} \mathrm{Med}$ J 293:1265-1267 